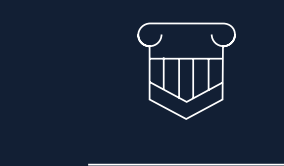

\title{
Capítulo II
}

\section{Educación jurídica en Colombia en modalidades a distancia y virtual: revisión normativa}

Elliot Parra Ávila1,2

\section{Presentación}

La consulta del Sistema Nacional de Información de la Educación Superior - SNIES (2018a) permite establecer que en Colombia se ofrecen en la actualidad 4.342 programas de pregrado profesional universitario. De ellos, "Administración de Empresas" es el más numeroso con 291 programas, seguido de "Contaduría Pública" con 239 programas y "Derecho" (incluye "Jurisprudencia" y "Derecho y Ciencias Políticas") con 192. Es decir, que los cinco (5) programas que más se ofrecen ${ }^{3}$ en el país suman 1.052 , y concentran el $24,2 \%$ de la oferta educativa superior en el nivel universitario, casi una quinta parte.

1 Agradezco a la abogada Nidia Robles Villabona (docente de la Universidad Santo Tomás) por sus contribuciones críticas.

\footnotetext{
2 Abogado y Magíster en Derecho de la Universidad Nacional de Colombia, Especialista en Derecho Procesal de la Universidad del Rosario y Master of Research del European University Institute. Docente Asociado de la Universidad Militar Nueva Granada y profesor universitario de pregrado y posgrado, par académico de Conaces y Conjuez del Tribunal Administrativo de Cundinamarca. ORCID: https://orcid.org/0000-0003-4659-7140. Correo electrónico: eliot. parra@unad.edu.co

3 Les siguen "Ingeniería de Sistemas" (incluida la denominación "Ingeniería Informática") con 169 programas e "Ingeniería Industrial" con 161 programas.
} 
Para los cinco programas más ofrecidos, el 13\% de los programas de Administración de Empresas (37), el 11\% de los programas de Contaduría Pública (27), el $9 \%$ de los programas de Ingeniería de Sistemas (16) y el $6 \%$ de los programas de Ingeniería Industrial (10), son impartidos en modalidades a distancia y virtuales (en adelante MaDVi) ${ }^{4}$. Para Derecho, el porcentaje de programas que se ofrece en modalidades a distancia o virtuales es de 0 , ya que el $100 \%$ de la oferta es presencial.

\section{Aspectos metodológicos}

Este trabajo pretende explorar las posibilidades que tiene la educación jurídica en MaDVi en Colombia, examinando dos aspectos: su regulación normativa y la aplicación administrativa. Para ello, serán analizadas las normas que regulan la educación superior en Colombia, desde que ella es considerada como una categoría autónoma, analizando el régimen de las modalidades no presenciales para relacionarlo con la regulación de los programas de Derecho (cualquiera sea su denominación) y otros similares (en especial en la última década), que han sido agrupados bajo la denominación "educación jurídica".

La búsqueda se centró en normas con rango de ley y actos administrativos que: a) autorizan las modalidades no presenciales, exclusivamente para ciertos programas y b) excluyen a los programas de Derecho de las modalidades no presenciales.

4 Las taxonomías en el derecho educativo colombiano presentan importantes problemas de coherencia. En algún momento se distinguió entre educación "no formal" e "informal" (en la Ley 115 de 1994, antes de la reforma introducida por la Ley 1064 de 2006) en contravía de lo que indica la lógica básica de oposiciones. A los niveles educativos se les denomina técnico profesional, tecnológico y profesional universitario; no obstante, los dos primeros pueden ser ofrecidos por universidades (lo que los convertiría en universitarios) y el tercero puede ser ofrecido por Instituciones Universitarias, Escuelas Tecnológicas e Instituciones Tecnológicas (lo que invalida el adjetivo de "universitarios"). Otro tanto sucede con las metodologías: a la metodología presencial se le opuso el término "a distancia" cuando estrictamente debería haber sido "no presencial". De esa confusión surgió la subdivisión administrativa de la metodología "a distancia" en las "modalidades" tradicional y virtual. 
La regulación normativa de la educación a distancia y virtual y su relación con los programas de Derecho $^{5}$

El antecedente de las regulaciones normativas de mayor relevancia para la educación no presencial ${ }^{6}$ es el Decreto 2412 (1982), para el que la Educación Superior Abierta y a Distancia era la que:

[...] se ofrece a quienes acrediten la calidad de bachilleres en cualquiera de sus modalidades y conduce a la obtención de títulos o a la acumulación de derechos académicos en las modalidades educativas de formación intermedia profesional, formación tecnológica, formación universitaria y formación avanzada o de postgrado (artículo 12).

Es decir, que desde las primeras expresiones de la educación superior a distancia7 esta ya abarcaba todos los niveles de formación y no distinguía entre áreas de conocimiento o programas en particular, consagrando la educación a distancia en igualdad de condiciones para todos los programas, instituciones y objetos de estudio.

El derecho constitucional (1991) vigente para escoger profesión tiene una limitación: la Ley podrá exigir títulos de idoneidad ${ }^{8}$. Esos títulos pueden ser otorgados tanto por el Estado como por los

5 Este documento fue escrito con anterioridad a la expedición del Decreto 1330 de 2019, sobre nuevas reglas de Registro Calificado (Ministerio de Educación Nacional, 2019).

6 Algunos estudios suelen iniciar la exposición sobre el tema con la Constitución Política de 1991 (Martín Calvo, 2018; Mesa Jiménez \& Forero Romero, 2016), pero se considera de gran importancia resaltar la existencia concreta de la educación superior a distancia con anterioridad al actual sistema de aseguramiento de la calidad.

7 Mediante la Ley 52 de 1981 se creó la "Unidad Universitaria del Sur de Bogotá". Posteriormente se denominó Universidad Nacional Abierta y a Distancia (Ley 396 de 1997).

8 "Toda persona es libre de escoger profesión u oficio. La ley podrá exigir títulos de idoneidad. [...]" (Art. 26, Constitución Política de Colombia). 
particulares ${ }^{9}$ que presten el servicio de educación ${ }^{10}$ en ejercicio de "las libertades de enseñanza, aprendizaje, investigación y cátedra" (Art. 27, C.P.) y de la autonomía universitaria ${ }^{11}$, todo ello bajo el control estatal ${ }^{12}$. Esto indica que en la prestación del servicio educativo concurren los derechos de los estudiantes y de los prestadores de dicho servicio, la obligación regulatoria del Estado y la obligación de esos tres actores para cumplir los fines constitucionales de la educación. El "servicio público de la Educación Superior", por su parte, se regula por la Ley 30 (1992) y esa situación especial fue reconocida por la Ley General de Educación (Ley 115 de 1994) que estableció posterior y taxativamente que la educación superior "es regulada por ley especial" (Art. 1).

Después de haber descrito los campos de acción de la educación superior (Art. 7) y sus niveles de formación (arts. 8-13), la Ley 30 de 1992 establece que "Las instituciones de Educación Superior podrán adelantar programas en la metodología de educación abierta y a distancia, de conformidad con la presente Ley" (Art. 15). De acuerdo con esto, la disposición que consagra la metodología a distancia no hace referencia a campo, programa ni nivel alguno, por lo que ha de entenderse como regla general y común a todos los campos y niveles de formación, con sus respectivos programas incluidos.

9 "Los particulares podrán fundar establecimientos educativos. La ley establecerá las condiciones para su creación y gestión. [...]" (Art. 68, Constitución Política de Colombia).

10 "La educación es un derecho de la persona y un servicio público que tiene una función social; con ella se busca el acceso al conocimiento, a la ciencia, a la técnica, y a los demás bienes y valores de la cultura [...]" (Art. 67, Constitución Política de Colombia).

11 "Se garantiza la autonomía universitaria. Las universidades podrán darse sus directivas y regirse por sus propios estatutos, de acuerdo con la ley. [...]" (Art. 69, Constitución Política de Colombia).

12 "[...] Corresponde al Estado regular y ejercer la suprema inspección y vigilancia de la educación con el fin de velar por su calidad, por el cumplimiento de sus fines y por la mejor formación moral, intelectual y física de los educandos [...]" (Art. 67, Constitución Política de Colombia). 
La Ley 1324 (2009), como parte del marco normativo del sector, es importante por establecer unas evaluaciones de resultado de la educación ("para medir el nivel de cumplimiento de sus objetivos y buscar el mejoramiento continuo" - Art. 1) denominadas "Exámenes de Estado", tanto para la educación media como para la superior. Más específicamente, se señala que:

Los "Exámenes de Estado" a los que se refieren los literales anteriores tendrán como propósito evaluar si se han alcanzado o no, y en qué grado, objetivos específicos que, para cada nivel o programa, según el caso, señalan las Leyes 115 de 1994 y 30 de 1992 y sus reglamentos, las que las modifiquen o complementen (Art. 7, literal b, inciso 3).

De esto se aprecia que la Ley que regula las pruebas de Estado establece distinciones en la medición de resultados por niveles de formación y por programas, pero no por metodologías. En este sentido, la evaluación de resultados de la educación superior en Colombia iguala a la metodología presencial con las MaDVi.

El Registro Calificado de programas, como el reconocimiento que hace el Estado del cumplimiento de las condiciones mínimas de calidad para su adecuado funcionamiento, fue introducido por la Ley 749 de 2003 y reglamentado por el Decreto 2566 de 2003. En esta norma, una vez más, dentro de la metodología a distancia se trata por igual a todos los programas en sus aspectos curriculares:

Los programas académicos de educación superior ofrecidos en la metodología de educación a distancia deberán demostrar que hacen uso efectivo de mediaciones pedagógicas y de las formas de interacción apropiadas que 
apoyen y fomenten el desarrollo de competencias para el aprendizaje autónomo y la forma como desarrollarán las distintas áreas y componentes de formación académica (Art. 4, inciso 5).

En cuanto a los medios educativos, el Decreto 2566 de 2003 establecía que la educación superior a distancia tenía las mismas funciones sustantivas que su homóloga presencial:

Para programas que se desarrollen en la metodología de educación a distancia, la institución deberá disponer de los recursos y estrategias propios de dicha metodología, a través de las cuales se atiende el acceso permanente de todos los estudiantes y profesores a la información, experimentación y práctica profesional, necesarias para adelantar procesos de formación, investigación y proyección social [...] (Art. 10, parágrafo).

Con base en el Decreto 2566 de $2003^{13}$ se expidieron varias resoluciones de "características específicas de calidad" para programas de pregrado, siendo de interés para este documento, aquellas resoluciones que regularon los programas a distancia y los programas de Derecho.

En este sentido, se encuentra la Resolución 2755 (2006), la cual definía la educación superior a distancia como:

[...] aquella metodología educativa que se caracteriza por utilizar ambientes de aprendizaje en los cuales se hace uso intensivo de diversos medios de información y comunicación y de mediaciones pedagógicas que permiten crear una dinámica de interacciones orientada al aprendizaje autónomo

13 El Decreto 2566 de 2003 estuvo vigente hasta el 25 de abril de 2008, cuando fue derogado por la Ley 1188 de 2008. No obstante, el 16 de junio de 2011, el Consejo de Estado declaró la nulidad del Decreto en la sentencia del expediente 11001-03-24-000-2007-00386-00 (2011). 
y abierto; superar la docencia por exposición y el aprendizaje por recepción, así como las barreras espacio-temporales y las limitaciones de la realidad objetiva mediante simulaciones virtuales; adelantar relaciones reales o mediadas y facilitar aprendizajes por indagación y mediante la colaboración de diversos agentes educativos (Art. 1).

Son tres las notas destacadas con relación a esta norma. En primer lugar, le asignaba a la educación superior a distancia la tarea de superar uno de los mayores problemas y más frecuentes críticas de la educación "tradicional": "superar la docencia por exposición y el aprendizaje por recepción"14. Segundo, aunque era reglamentaria de un decreto sobre educación "a distancia", incorporó por vez primera la noción de virtualidad en el ordenamiento jurídico educativo colombiano ${ }^{15}$. Tercero, hizo expreso el hecho de que un mismo programa pudiera ser ofrecido tanto en metodología presencial como a distancia y además los igualó en cuanto a su peso en créditos:

En aquellas instituciones que ofrezcan el mismo programa bajo la metodología presencial y a distancia, el número de créditos de los programas a distancia será igual al de los programas presenciales (Art. 3, Resolución 2755 de 2006).

Por su parte, la Resolución 2768 (2003) definió las características específicas de calidad para los programas de pregrado universitario en derecho y en ella no se hizo referencia alguna, en ningún sentido, a las metodologías bajo las cuales podían ser ofrecidos ${ }^{16}$.

14 La crítica sobre el aprendizaje por recepción sigue vigente (García Jiménez \& Lorente García, 2017) y tiene abundante literatura (Aranda Parra \& Parra Muñoz, 2015)

15 En esta norma también consagraba que (sobre medios educativos) los programas debían contar con "laboratorios físicos o virtuales, de apoyo a la actividad académica e investigativa, señalando las estrategias para atender a estudiantes" (literal 3, Art. 5, Resolución 2755 de 2006).

16 Teniendo en cuenta que las resoluciones de características específicas fueron expedidas por el Ministerio de Educación Nacional con base en la orden contenida en los artículos 1 y 4 del Decreto 2566 de 2003, y que este fue declarado nulo. 
La parte de la Ley 749 de 2002 sobre programas técnicos y tecnológicos que establecía los registros calificados para los programas universitarios fue declarada inexequible por la sentencia C-852 (2005) por violar la unidad de materia, razón por la que el Congreso de la República expidió la Ley 1188 (2008) que regula los registros calificados de programas de educación superior en general. Como era de esperarse, en dicha Ley tampoco hay referencias a las metodologías bajo las que pueden ser ofrecidos los programas académicos, pero como hecho por destacar se incorporó como condición de los medios educativos para todos los programas en el país, una que hasta ese momento era exclusiva de los programas a distancia: "El uso adecuado y eficiente de los medios educativos de enseñanza que faciliten el aprendizaje y permitan que el profesor sea un guía y orientador y el estudiante sea autónomo y participante"17 (Art. 2, num. 8) ${ }^{18}$

El Decreto Reglamentario de la Ley 1188 de 2008 fue el Decreto 1295 de 2010. Por su naturaleza, esta reglamentación entró en detalles al regular los programas en MaDVi, al exigirles como condiciones para obtener el registro calificado: idoneidad de sus docentes en las metodologías no presenciales y capacitación en Tecnologías de la Información y la Comunicación, TIC, (Art. 5.7.1.4.); indicar el proceso de diseño, gestión, producción, distribución y uso de materiales y recursos y para los programas virtuales: el $15 \%$ de los cursos disponibles en plataforma tecnológica apropiada, con la infraestructura de conectividad y

Es claro que todas aquellas perdieron fuerza ejecutoria bajo las reglas del Código Contencioso Administrativo vigente en esos años, que contemplaba en su artículo 66 que los actos administrativos "perderán su fuerza ejecutoria en los siguientes casos: [...] 2. Cuando desaparezcan sus fundamentos de hecho o de derecho $[\ldots]^{\prime \prime}(1984)$.

17 En efecto, el asunto de la autonomía del estudiante se consideraba propio de la educación a distancia, tal como consta en el Decreto 2566 de 2003 sobre demostrar el uso de "formas de interacción apropiadas que apoyen y fomenten el desarrollo de competencias para el aprendizaje autónomo" (Art. 4) y en la Resolución 2755 de 2006 al referirse a "una dinámica de interacciones orientada al aprendizaje autónomo" (Art. 1), ambas mencionadas y resaltadas anteriormente.

18 En el mismo sentido, el Decreto 1001 (2006) sobre programas de posgrado al ordenarles "demostrar que hacen uso efectivo de mediaciones y de formas de interacción apropiadas que apoyen y fomenten el desarrollo de competencias para el trabajo autónomo del estudiante" (Art. 13, num. 3). 
las herramientas metodológicas necesarias para su desarrollo, así como las estrategias de seguimiento, auditoría y verificación de esta (Art. 5.8); indicar que se dispone de las condiciones de infraestructura y de medios educativos en centros de tutoría, de prácticas, clínicas o talleres, de ser requerido (Art. 5.9), mecanismos de selección, inducción a la modalidad, seguimiento y acompañamiento a los estudiantes por parte de los tutores o consejeros (Art. 6.1) y garantías de que los estudiantes podrán participar del bienestar universitario (Art. 6.5). Es de anotar que se ordenó la autorización previa por parte del Ministerio de Educación para la "Adopción de la modalidad virtual en un programa a distancia" (Art. 42.7).

Es de particular interés que el Decreto 1295 (2010) haya contemplado que los programas virtuales puedan tener una parte de presencialidad de máximo el $20 \%$ del total de las actividades académicas. Consecuentemente, si esas actividades de formación requieren la realización presencial de prácticas, clínicas o talleres o la presencia de los estudiantes en centros de tutoría, esos lugares quedan sujetos a condiciones de calidad, estabilidad y publicidad (Art. 16 a 19).

El Decreto 1075 (2015) buscó unificar todo el régimen jurídico de la educación colombiana, desde preescolar hasta superior. Para esos efectos incorporó principalmente, en materia de educación superior, los decretos 1212 de 1993, 860 y 2216 de 2003, 1001 de 2006, 4790 de 2008, 1295 de 2010, 2545 de 2014 y 2450 de 2015, entre otros. Las recientes reformas, en especial las relacionadas con programas de licenciaturas y enfocados a la educación, han afectado a los programas en MaDVi de dichas áreas (Mesa \& Forero, 2016), pero no a través de la regulación directa de las modalidades, sino por la regulación de sus actividades presenciales. En este sentido, la regulación de programas que se ofrecen en varias metodologías los afecta de forma diversa (Mera, 2018); sin embargo, no hay evidencia de que sea una regulación especial para alguna de ellas. 
De lo expuesto, se puede concluir que a) no existe en Colombia limitación jurídica general para que ciertos campos, áreas, niveles o programas de educación superior sean ofrecidos en determinadas metodologías; b) las normas especiales sobre educación superior a distancia y virtual tampoco hacen referencia a campos, áreas, niveles o programas a las que estén dirigidas: son normas especiales por su objeto, pero generales respecto de los programas; c) las normas especiales sobre programas de derecho tampoco hacen referencia a las metodologías en la que puedan ser ofrecidos y d) las normas especiales surgidas recientemente están dirigidas a programas totalmente diferentes a los jurídicos. En resumen, no hay posibilidad de que el sistema normativo colombiano pueda ser interpretado en el sentido de que existe algún fundamento jurídico para impedir que un programa de pregrado universitario en Derecho sea ofrecido en MaDVi.

\section{La aplicación administrativa de las normas sobre educación a distancia y virtual y su relación con los programas de derecho}

Se ha concluido que no existe razón jurídica alguna que explique la inexistencia de programas de pregrado universitario de derecho en Colombia, a distancia o virtuales. Este hecho resulta curioso, si se considera que Derecho es el único programa dentro de los diez (10) más ofrecidos sin oferta en esta modalidad ${ }^{19}$.

En la primera parte de este texto se examinaron las normas de carácter general (leyes, decretos) que, por supuesto, incluyen actos administrativos. En complemento, en este apartado se

19 Recapitulando, los diez programas más ofrecidos son, en orden descendente, Administración de Empresas, Contaduría Pública, Derecho (incluye Jurisprudencia y Derecho y Ciencias Políticas), Ingeniería Industrial, Ingeniería de Sistemas (incluye Ingeniería Informática), Piscología, Ingeniería Electrónica, Economía, Ingeniería Civil e Ingeniería Ambiental. 
pretende examinar la regulación administrativa concreta, su aplicación, esto es, lo que ya no depende de leyes del Congreso o reglamentos del presidente, sino de la gestión administrativa cotidiana que hace el Ministerio de Educación Nacional.

Una nota preliminar de carácter metodológico tiene que ver con el acceso a la información sobre el otorgamiento o la negación de los registros calificados. La ley 1188 de 2008 estableció el carácter reservado de la información que las Instituciones de Educación Superior -IES- proporcionan en los procesos de registro calificado y ordenó al Ministerio de Educación Nacional publicar en el Sistema Nacional de Información de la Educación Superior, SNIES, "las decisiones favorables de los correspondientes procesos de evaluación" (Art. 4). Esto implica, sin discusión, que las decisiones desfavorables sobre registros calificados no deben ser publicadas en este sistema.

No obstante, subsiste la duda sobre la posibilidad de acceder, en caso de ser solicitados por derecho de petición, a los actos administrativos que niegan los registros calificados. Un argumento en pro de la entrega a un peticionario de las resoluciones desfavorables es que la reserva pesa sobre la información que las instituciones proporcionan en el proceso, no sobre los actos administrativos que los resuelven. Un argumento en pro de la reserva de las resoluciones desfavorables es que, en esos actos administrativos, en la parte motiva, suelen aparecer plasmados o transcritos los datos que las instituciones proporcionaron a la sala de Comisión Nacional Intersectorial de Aseguramiento de la Calidad de la Educación Superior -Conaces- ${ }^{20}$ durante el proceso $\mathrm{y}$, en ese sentido, las resoluciones desfavorables contendrían

20 La Comisión Nacional Intersectorial de Aseguramiento de la Calidad de la Educación Superior es un órgano de asesoría y coordinación que "se encarga de la evaluación del cumplimiento de los requisitos para la creación de instituciones de educación superior, su cambio de carácter académico, redefinición, creación de seccionales y reconocimiento como universidades, así como también le corresponde evaluar que los programas académicos cumplan con las condiciones de calidad para su oferta y desarrollo, y emitir el respectivo concepto sobre la procedencia del otorgamiento o renovación del registro calificado" (Ministerio de Educación Nacional, 2015). 
parte de esa información proporcionada por las instituciones sobre la que pesa la reserva. Teniendo en cuenta la dinámica del sector educativo superior, es difícil determinar si el Ministerio de Educación Nacional ha sido puesto a prueba en esta materia y cuál ha sido su posición.

Dicho lo anterior, y ante la dificultad para acceder a los motivos que han llevado al Ministerio de Educación Nacional a negar el otorgamiento de registros calificados, el examen de la forma en que se han interpretado las normas de registro calificado y la realidad de la oferta educativa superior actual puede dar razones sobre las posibilidades de los programas de Derecho en MaDVi.

En 2003, el Ministerio de Educación Nacional publicó los Lineamientos de Calidad para la verificación de las condiciones de calidad de los programas virtuales y a distancia (Castillo, 2013). Los lineamientos son el documento en el que se intentó justificar la distinción entre las "modalidades" de "a distancia tradicional" y "a distancia virtual" que compondrían la categoría superior de "metodología a distancia" (2013, p. 5) Aunque esa terminología no es de la Ley 30 de 1992, en adelante se asumirá en la medida en que tanto el SNIES como los procesos de verificación de condiciones que se gestionan a través del Sistema de Aseguramiento de la Calidad de la Educación Superior, SACES, la utilizan y se ha impuesto por la praxis cotidiana de los sujetos del sistema (i.e. instituciones, pares, comisionados, funcionarios), no por su corrección lógica y categorial. Aparte de lo dicho, los lineamientos se muestran como un documento técnico acorde con la tecnología disponible en el momento de su elaboración.

Otra de las innovaciones administrativas que el Ministerio de Educación Nacional ha introducido en los procesos de registro calificado es denominar al documento que expone las condiciones de calidad "documento maestro". Según Conaces, la guía para la elaboración del documento maestro de registro calificado surgió 
"como resultado de la experiencia de más de 10 años en la evaluación de solicitudes relacionadas con el Registro Calificado" (Subdirección de Aseguramiento de la Calidad de Educación Superior, 2016, p. 1). La guía, siguiendo el Decreto 1075 de 2015, no hace distinción alguna sobre campos, áreas, niveles o programas que deban o puedan ser ofrecidos en modalidades a distancia o virtual.

En armonía con el establecimiento de una guía para las instituciones, con el apoyo del Instituto Colombiano de Normas Técnicas y Certificación (Icontec), el Ministerio de Educación Nacional elaboró la Norma Técnica para Pares Académicos NTPA-MEN (2016). Esta busca establecer lineamientos para la verificación de las condiciones de calidad de programas académicos de educación superior. En la NTPA, aparte de introducir el neologismo "créditos virtualizados" (para programas virtuales) ${ }^{21}$, tampoco se hace referencia alguna a los alcances de las MaDVi en función de campos, áreas, niveles o programas.

Luego se establece que ninguno de los reglamentos del Ministerio de Educación Nacional permite identificar algún tipo de sesgo hacia el ofrecimiento de programas de pregrado profesional de Derecho en modalidades a distancia y virtual. Es entonces en la praxis en la que se deben encontrar razones para explicar la realidad de dichos programas. Al respecto, la literatura colombiana es prácticamente inexistente ${ }^{22}$ (Ver Tabla N. ${ }^{\circ} 7$ ).

\begin{tabular}{|c|c|c|}
\hline Descriptor & $\begin{array}{c}\text { Obras } \\
\text { sobre Colombia }\end{array}$ & $\begin{array}{c}\text { Obras } \\
\text { sobre otros países }\end{array}$ \\
\hline $\begin{array}{c}\text { Enseñanza a distancia del } \\
\text { derecho }\end{array}$ & 0 & 2 \\
\hline $\begin{array}{c}\text { Enseñanza virtual del } \\
\text { derecho }\end{array}$ & 0 & 8 \\
\hline
\end{tabular}

21 La denominación de la Ley 1188 de 2008 es la de "actividades académicas" y son ellas las que se virtualizan, no los créditos que expresan su cantidad de trabajo académico (Art. 2.5.3.2.4.1 del Decreto 1075 de 2015).

22 Consulta en Google Scholar fijando como año de inicio de la búsqueda 2010, en razón a la expedición del Decreto 1295 de 2019, solo páginas en español, excluyendo patentes y citas y con verificación del contenido de cada obra hallada. 


\begin{tabular}{|c|c|c|}
\hline $\begin{array}{c}\text { Educación jurídica a } \\
\text { distancia }\end{array}$ & 0 & 0 \\
\hline Educación jurídica virtual & 0 & 0 \\
\hline
\end{tabular}

Tabla N. ${ }^{\circ}$ 7. Resultados de Google Scholar para descriptores

Colombianos y extranjeros

Fuente: elaboración propia

Ante la ausencia de un debate académico concreto sobre la aptitud de las metodologías a distancia y virtual para la educación jurídica ${ }^{23}$, se propone considerar hipotéticamente las posibles objeciones que podrían surgir frente a dicha aptitud para contrastarlas con la actual oferta de programas (en todos los niveles) del Núcleo Básico de Conocimiento, $\mathrm{NBC}$, : derecho y afines.

Una primera objeción podría consistir en afirmar que para ofrecer programas de pregrado profesional en derecho se requiere que las instituciones tengan una doble experticia: en Derecho y en MaDVi. Esa objeción presenta al menos dos problemas: a) implica que se debe haber ofrecido un programa en metodología presencial para poder ofrecerlo en MaDVi, estableciendo un prerrequisito ajeno a la normativa y la experiencia de los programas que han sido ofrecidos por vez primera a distancia o virtuales y b) omite el hecho de que hay instituciones que no ofrecen programas presenciales. Frente a esto, la evidencia indica que existen ciento diez instituciones que ofrecen el programa de Derecho y también programas en MaDVi; es decir, que estarían en situación de proponer programas de pregrado universitario de Derecho a distancia o virtuales. ¿Es racional admitir que ninguna de ellas ha logrado proponer uno viable?

Una segunda objeción consistiría en defender que la virtualidad solo sería apta para niveles de formación superiores (i.e. especialización, maestría y doctorado). A esta objeción no se

23 Se está ante un caso más de un fenómeno general del Derecho que ha señalado García (2010) "No es una sorpresa si, bajo estas condiciones, en Colombia se habla de crisis del derecho. Lo que sorprende es que se hable tan poco" (p. 23). 
le puede responder con la lógica de a maiori ad minus, ya que las diferencias entre niveles son cualitativas, pero sí se puede responder con otros argumentos. Los dos problemas de esta objeción están en su base normativa: a) la Ley 30 de 1992 define las especializaciones como aquellas "que se desarrollan con posterioridad a un programa de pregrado y posibilitan el perfeccionamiento en la misma ocupación, profesión, disciplina o áreas afines o complementarias" (Art. 10). Si el perfeccionamiento que recae sobre un objeto puede hacerse a distancia o virtual, no hay razón para sostener que el estudio inicial, básico o general de dicho objeto tenga que hacerse necesariamente en otra metodología. El asunto, como se dijo, no tiene que ver con el menos y el más, sino con lo principal y lo accesorio. Y, b) la Ley 30 de 1992 establece que:

Las maestrías buscan ampliar y desarrollar los conocimientos para la solución de problemas disciplinarios, interdisciplinarios o profesionales y dotar a la persona de los instrumentos básicos que la habilitan como investigador en un área específica de las ciencias o de las tecnologías o que le permitan profundizar teórica y conceptualmente en un campo de la filosofía, de las humanidades y de las artes (Art. 12).

Si se retoma que la única condición de calidad en la que el uso de las TIC es obligatorio para los programas presenciales es la de Investigación (Decreto 1075 de 2015, Art. 2.5.3.2.2.1., num. 5.2), se puede afirmar que las maestrías son programas en los que se amplía (una) parte de la formación básica del pregrado (el todo), máxime si se considera que las pruebas Saber PRO (Examen de calidad de la educación superior) para Derecho evalúan la competencia específica de investigación jurídica, que se presume ya desarrollada por el estudiante con anterioridad a la maestría. 
La evidencia indica que, por los 192 programas de pregrado en Derecho ofrecidos en Colombia, hay 769 posgrados (i.e. 609 especializaciones universitarias, 145 maestrías y 15 doctorados) y de ese total, existen seis especializaciones y dos maestrías en modalidades a distancia y virtual (creadas en los años 2007, $2009,2013,2015$ y 2016), de forma tal que muy recientemente se ha aceptado la modalidad a distancia y virtual para posgrados, con las implicaciones expuestas.

Como tercera objeción se podría esgrimir la sobreoferta del programa de Derecho. En este punto debe ir por delante que cualquier juicio sobre la sobreoferta es un juicio acerca de la metodología presencial exclusivamente, que representa el $100 \%$ de la oferta; pero como homogeneidad no significa necesariamente sobreoferta, se deben examinar algunas estadísticas (Ver Ilustración N. ${ }^{\circ} 1$ ).

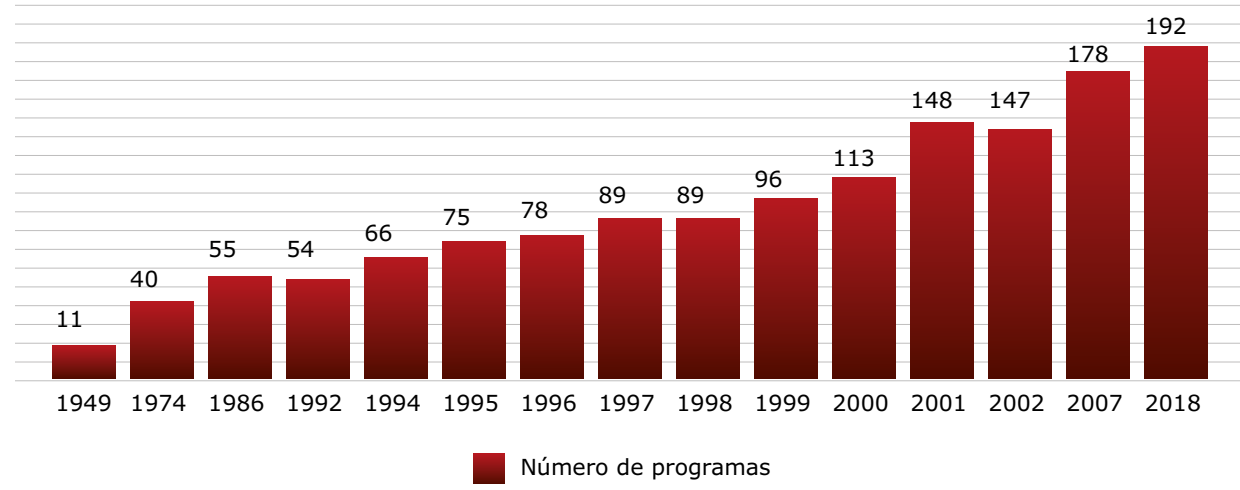

Ilustración N.* 1: Número de programas de derecho en Colombia 1949-2018 Fuente: elaboración propia con base en Salamanca, 2010, p. 113.

De acuerdo con lo anterior, el número de programas se ha incrementado a partir de 1992, lo que coincide con el notable aumento de los cupos en programas de pregrado universitario de derecho entre 1985 y 2000, que "poco o nada tiene que ver con el aumento poblacional antes mencionado, pues mientras la población aumenta un 30,8\%, la oferta en educación jurídica lo hace en un 197,1\%" (Sánchez, 2010, p. 232). Por tanto, el 
aumento de programas y de cupos a un ritmo muy superior al del incremento poblacional dan como resultado la sobreoferta de profesionales, ya que como lo ha expuesto la Organización para la Cooperación y el Desarrollo Económicos, OCDE, el 45\% de los graduados en Colombia de educación superior están ubicados en el campo de "Negocios, Administración y Derecho", cuando el promedio OCDE respectivo es de 24\% (2017, p. 72), dato armónico con el de oferta de programas expuesto al inicio de este texto. Si el problema es de sobreoferta, esta debería ser controlada respecto de todas las metodologías, no solamente respecto de las MaDVi.

La cuarta objeción consistiría en que la naturaleza de las competencias del Derecho hace inviable ofrecer dicho programa en MaDVi. La idea de los Núcleos Básicos de Conocimiento (NBC) en los que están organizadas las áreas de conocimiento en el SNIES, y que afectan la composición de las salas de Conaces (Ministerio de Educación Nacional, 2018b), puede servir para examinar dicha objeción. A esta objeción se le pueden proponer una respuesta fuerte y una respuesta débil. La respuesta fuerte recuerda que la pertenencia al mismo $\mathrm{NBC}^{24}$ implica necesariamente similitudes en los objetos de conocimiento de los programas. Adicionalmente, las competencias específicas de Derecho (i.e. comunicación jurídica, gestión del conflicto e investigación jurídica) ${ }^{25}$ pueden ser ejecutadas a través de medios digitales o de forma presencial. Por oposición, los programas técnicos y tecnológicos existentes, enfocados en el desarrollo de competencias profesionales, educan para un desempeño laboral que difícilmente se puede realizar a distancia o virtualmente.

24 Inexplicablemente dos de los programas de Derecho están clasificados en el NBC "Geografía, Historia" y otros cinco programas ni siquiera tienen Área de Conocimiento ni NBC.

$25 \mathrm{El}$ hecho de que estas tres áreas sean las competencias específicas que el Instituto Colombiano para la Evaluación de la Educación - Icfes evalúa del programa de Derecho, no quiere decir que ellas sean exclusivamente un sistema de evaluación de resultados. Son un sistema de competencias de la formación, ya que esas tres competencias fueron las que la Asociación Colombiana de Facultades de Derecho, Acofade, estableció como en sus lineamientos sobre programas de derecho (AA. VV., 2017). 
EI NBC "Derecho y afines" está compuesto por los siguientes programas de pregrado (219) ${ }^{26}$ :

-Técnicos profesionales en Balística, Criminalística, Fotografía Forense, Topografía Forense y "Judicial".

- Tecnológicos en Criminalística, Gestión Judicial, Investigación Criminal, Investigación Criminalística, Investigación de Accidentes de Tránsito, Investigación Judicial, Procedimientos Judiciales y Promoción y Aplicación del DIH y el DIDH.

- Universitarios de Criminalística, Derecho, Gobierno e Investigación Criminal.

De ellos, excluyendo Derecho, se ofrecen en total treinta y cuatro programas, ocho de los cuales $(23,5 \%)$ están en MaDVi ${ }^{27}$ : Técnico Profesional Judicial (virtual); Tecnologías en Criminalística, Gestión Judicial, Investigación de Accidentes de Tránsito y Procedimientos Judiciales (a distancia) y Universitario de Investigación Criminal (virtual y también presencial).

Más allá de que algunos programas del NBC analizado no cumplen con la exigencia legal de "adoptar denominaciones que correspondan con las competencias propias de su campo de conocimiento" (Decreto 1075 de 2015, Art. 2.5.3.2.2.1., num. 5.1), es notable que mientras parece haber una fuerte resistencia a la aprobación de programas de pregrado profesional universitario de Derecho a distancia o virtuales, esas modalidades son muy extendidas si la formación es técnico profesional o tecnológica (duplicando el promedio del total) ${ }^{28}$. No obstante, esos programas implican desempeños laborales casi que necesariamente presenciales, en la medida en que

26 También inexplicablemente en el NBC "derecho y afines" aparecen como pregrados una Especialización, dos programas de Psicología y uno de Comunicación Social.

27 La aparente diferencia de cifras se explica en la anterior nota al pie de página.

28 El total de programas de pregrado es de 7.267 , de los cuales 842 se ofrecen en MaDVi, es decir el $11,6 \%$. 
tratan sobre competencias fuertemente mediadas por principios "presenciales" del derecho procesal general como los de oralidad e inmediación (Ley 1564 de 2012) y del derecho procesal penal como los de oralidad, contradicción, inmediación y concentración (Ley 906 de 2004).

La respuesta débil está asociada con la estructura general de la oferta educativa y profesional colombiana: ante las dificultades para controlar la oferta educativa jurídica mediante los registros calificados y las acreditaciones de alta calidad, el Estado ha optado por controlar la oferta de graduados que pueden ejercer la profesión mediante el Examen de Estado que realizará el Consejo Superior de la Judicatura (Ley 1905 de 2018). Si se ha optado por someter al estudiante a un examen para poder graduarse (Saber PRO) y al egresado a otro para poder litigar (Examen de Estado), es porque se ha optado por un modelo de calidad de medición de resultado (fines), lo que implicaría mayor flexibilidad respecto de los medios, tal como sucede en el caso del bachillerato, en el que el peso del puntaje de la prueba Saber 11 se impone en la práctica sobre cualquier otro criterio acerca de la calidad de la formación recibida. Esta segunda respuesta a la objeción de viabilidad sería más adecuada para responder a una objeción de calidad, pero se ha dicho que ese debate sobre la calidad potencial de programas de pregrado universitario de derecho está pendiente de ser adelantado en Colombia y, en ese orden de ideas, el presente trabajo ha buscado acotarlo y presentarlo. 


\section{Conclusiones}

La regulación normativa de la educación superior colombiana permite que existan programas jurídicos en MaDVi. Luego, considerando que existen 110 instituciones con experticia, tanto en derecho como en MaDVi, se justifica indagar sobre las causas de la inexistencia de programas de pregrado universitario en Derecho ofrecidos en las modalidades de educación a distancia y virtual. No obstante, no parecen existir razones fuertes para justificar la existencia de programas en MaDVi de posgrado, pero no de pregrado universitario. Al respecto, la evidencia revela que los programas jurídicos técnicos y tecnológicos en MaDVi tienen una participación en su Núcleo Básico de Conocimiento que dobla el promedio nacional total de programas, aunque su objeto de estudio y de desempeño profesional puede llegar a ser más presencial que el de otros programas jurídicos. Así, la sobreoferta de programas de pregrado universitario ha sido generada exclusivamente desde la metodología presencial y existe un programa profesional universitario del NBC derecho y afines (Investigación criminal) que se ofrece tanto presencial como en MaDVi, lo que implica que este nivel ya ha sido abierto para esas modalidades.

\section{Referencias bibliográficas}

AA. VV. (2017). Lineamientos técnicos para los programas de Derecho en Colombia. Medellín: Asociación Colombiana de Facultades de Derecho, ACOFADE. Corporación Universitaria Remington. EPA.

Aranda, V., \& Parra, H. (2015). Tiempos críticos y pedagogías críticas. Compendio de las teorías educativas críticas. Diálogos educativos, 14(28), p. 119-134.

Castillo, M. (2013). Lineamientos de calidad para la verificación de las condiciones de calidad de los programas virtuales y a distancia. Bogotá D.C.: Ministerio de Educación Nacional y Convenio Andrés Bello. 
Congreso de la República de Colombia (1991). Constitución Política. Recuperado de: http://www.secretariasenado.gov.co/index.php/ leyes-y-antecedentes/constitucion-y-sus-reformas.

Congreso de la República de Colombia (1981). Ley 52, porla cual se crea la Unidad Universitaria del Sur de Bogotá y se dictan otras disposiciones. Recuperado de http://www.suin-juriscol.gov.co/clp/contenidos.dll/ Leyes $/ 1606318$ ?fn=document-frame.htm $\$ f=$ templates $\$ 3.0$.

Congreso de la República de Colombia (1992). Ley 30, por la cual se organiza el servicio público de la Educación Superior. Recuperado de:http://www.secretariasenado.gov.co/senado/basedoc/ ley_0030_1992.html\#1.

Congreso de la República de Colombia (1994). Ley 115, por la cual se expide la ley general de educación. Recuperado de http://www. secretariasenado.gov.co/senado/basedoc/ley_0115_1994.html.

Congreso de la República de Colombia (1997). Ley 396, por la cual se transforma la Unidad Universitaria del Sur de Bogotá, en Universidad Nacional Abierta y a Distancia UNAD - y se dictan otras disposiciones. Recuperado de: http://www.secretariasenado.gov.co/senado/basedoc/ ley_0396_1997.html.

Congreso de la República de Colombia (2003). Ley 749, por la cual se organiza el servicio de la educación superior en las modalidades de formación técnica profesional y tecnológica. Recuperado de: http:// www.secretariasenado.gov.co/senado/basedoc/ley_0749_2003.html.

Congreso de la República de Colombia (2004). Ley 906, por la cual se expide el Código de Procedimiento Penal. Recuperado de: http://www. secretariasenado.gov.co/senado/basedoc/ley_09060_204a.html.

Congreso de la República de Colombia (2008). Ley 1188, por la cual se regula el registro calificado de programas de educación superior $y$ se dictan otras disposiciones. Recuperado de: http://www. secretariasenado.gov.co/senado/basedoc/ley_1188_2008.html.

Congreso de la República de Colombia (2009). Ley 1324, por la cual se fijan parámetros y criterios para organizar el sistema de evaluación de resultados de la calidad de la educación, se dictan normas para el fomento de una cultura de la evaluación, en procura de facilitar la inspección y vigilancia del Estado y se transforma el ICFES. Recuperado de: http://www.secretariasenado.gov.co/senado/ basedoc/ley_1324_2009.html. 
Congreso de la República de Colombia (2012). Ley 1564, por medio de la cual se expide el Código General del Proceso y se dictan otras disposiciones. Recuperado de: http://www.secretariasenado.gov.co/ senado/basedoc/ley_1564_2012.html.

Congreso de la República de Colombia (2018). Ley 1905, por la cual se dictan disposiciones relacionadas con el ejercicio de la profesión de abogado. Recuperado de: http://www.funcionpublica.gov.co/eva/ gestornormativo/norma.php?i=87186.

Consejo de Estado (2011). Sentencia del expediente 11001-03-24000-2007-00386-00, Acción de nulidad contra el Decreto 2566 de 2003. C.P. María Elizabeth García González.

Corte Constitucional (2005). Sentencia C-852. Demanda de inconstitucionalidad contra el artículo 8 (parcial) de la ley 749 de 2002 «por la cual se organiza el servicio público de la educación superior en las modalidades de formación técnica profesional y tecnológica». M.P. Rodrigo Escobar Gil.

García, E., \& Lorente, R. (2017). De receptor pasivo a protagonista activo del proceso de enseñanza-aprendizaje: Redefinición del rol del alumnado en la Educación Superior. Opción, 33(84), p. 120-153.

García, M. (2010). Los abogados en Colombia. Bogotá D.C.: Universidad Nacional de Colombia.

Martin, J. F. (2018). Calidad educativa en la educación superior colombiana: Una aproximación teórica. Sophia-Educación, 14(2), 4-14. Recuperado de: http://dx.doi.org/10.18634/sophiaj.14v.2i.799

Mera, D. (2018, febrero 2). El problema postergado de las licenciaturas. elespectador.com. Recuperado de: https://www.elespectador. com/opinion/el-problema-postergado-de-las-licenciaturascolumna-736964

Mesa, F. \& Forero, A. (2016). Las TIC en la normativa para los programas de educación superior en Colombia. Praxis \& Saber, 7(14), 91-113. Recuperado de: https://doi.org/10.19053/22160159.5219

Ministerio de Educación Nacional. (2003). Resolución 2768, Por la cual se definen las características específicas de calidad para los programas de pregrado en Derecho. Recuperado de: https://www. mineducacion.gov.co/1621/articles-86421_Archivo_pdf.pdf. 
Ministerio de Educación Nacional. (2006). Resolución 2755, Por la cual se definen las características específicas de calidad para la oferta y desarrollo de los programas académicos en la metodología a distancia. Recuperado de: https://normograma.info/men/docs/pdf/ resolucion_mineducacion_2755_2006.pdf.

Ministerio de Educación Nacional. (2015). Salas de Conaces [Sistema de Aseguramiento de la Calidad de la Educación Superior]. Recuperado 19 de agosto de 2018, de Sistemas de Información. Recuperado de: https://www.mineducacion.gov.co/sistemasdeinformacion/1735/ article-227123.html

Ministerio de Educación Nacional. (2016, diciembre 20). Norma Técnica para Pares Académicos NTPA-MEN. Ministerio de Educación Nacional.

Ministerio de Educación Nacional. (2018a). Sistema Nacional de Información de la Educación Superior SNIES. Recuperado de: https:// snies.mineducacion.gov.co/consultasnies/programa\#.

Ministerio de Educación Nacional. (2018b). Resolución 10414, Por la cual se reorganiza la Comisión Nacional Intersectorial de Aseguramiento de la Calidad de la Educación Superior (CONACES), se adopta el reglamento para el funcionamiento de sus Salas de Revisión y Consulta, de Evaluación y de Coordinadores, y se derogan las Resoluciones 14830 de 2016 y 3179 de 2017. Recuperado de: https://www.mineducacion. gov.co/1759/w3-article-370916.html.

Ministerio de Educación Nacional (2019, julio 25). Decreto 1330, por el cual se sustituye el capítulo 2 y se suprime el Capítulo 7 del Título 3 de la Parte 5 del Libro 2 del decreto 1075 de 2015 - Único Reglamentario del Sector Educación". Recuperado de https://www.mineducacion. gov.co/1759/articles-387348_archivo_pdf.pdf

Organization for Economic Co-operation and Development. (2017). Education at a Glance 2017: OECD indicators. Recuperado de: http:// dx.doi.org/10.1787/eag-2017-en

Presidencia de la República (1982). Decreto 2412, por el cual se reglamenta, dirige e inspecciona la Educación Abierta y a Distancia y se crea el Consejo de Educación Abierta y a Distancia. Recuperado de: https://normograma.info/men/docs/decreto_2412_1982.htm.

Presidencia de la República (1984). Decreto 1, por el cual se reforma el Código Contencioso Administrativo. 
Presidencia de la República (2003). Decreto 2566, por el cual se establecen las condiciones mínimas de calidad y demás requisitos para el ofrecimiento y desarrollo de programas académicos de educación superior y se dictan otras disposiciones. Recuperado de: https:// www.mineducacion.gov.co/1621/articles-86425_Archivo_pdf.pdf.

Presidencia de la República (2006). Decreto 1001, por el cual se organiza la oferta de programas de posgrado y se dictan otras disposiciones. Recuperado de: http://www.alcaldiabogota.gov.co/sisjur/normas/ Norma1.jsp? $\mathrm{i}=19974$.

Presidencia de la República (2010). Decreto 1295, por el cual se reglamenta el registro calificado de que trata la Ley 1188 de 2008 y la oferta y desarrollo de programas académicos de educación superior. Recuperado de: http://www.funcionpublica.gov.co/eva/ gestornormativo/norma.php?i=39363.

Presidencia de la República (2015). Decreto 1075, por medio del cual se expide el Decreto Único Reglamentario del Sector Educación. Recuperado de http://www.suin-juriscol.gov.co/viewDocument. asp?id=30019930.

Salamanca, F. (2010). Educación legal en Colombia. Análisis cuantitativo desde una perspectiva histórica. En M. García Villegas, Los abogados en Colombia (pp. 103-143). Bogotá D.C.: Universidad Nacional de Colombia.

Sánchez, S. (2010). Análisis estadístico de la oferta y la demanda de educación jurídica en Colombia. En M. García Villegas, Los abogados en Colombia (pp. 225-255). Bogotá D.C.: Universidad Nacional de Colombia.

Subdirección de Aseguramiento de la Calidad de Educación Superior. (2016, mayo 31). Guía para la elaboración del Documento Maestro de Registro Calificado M-DS-MA-IA-01-01. Ministerio de Educación Nacional. 